\title{
Изоэлектронные центры кислорода и проводимость кристаллов CdS в сравнении с PbS
}

\author{
(C) Н.К. Морозова, Б.Н. Мирошников \\ Национальный исследовательский университет „Московский энергетический институт“, \\ 111250 Москва, Россия \\ Институт нанотехнологий микроэлектроники РАН (ИНМЭ РАН), \\ 119991 Москва, Россия \\ E-mail: MorozovaNK@mail.ru
}

(Получена 21 декабря 2016 г. Принята к печати 26 декабря 2016 г.)

Влияние кислорода на электрические свойства $\mathrm{PbS}$ широко известно. Целью данной работы было сопоставление и обнаружение этого явления в монокристаллах $\mathrm{CdS}(\mathrm{O})$, детально исследованных нами ранее. Эксперименты проведены на монокристаллах $\mathrm{CdS}$ с известной концентрацией кислорода, отклонением от стехиометрии и определенным набором собственных точечных дефектов. Они позволили подтвердить результаты, описанные для $\mathrm{PbS}$, и выяснить их природу. Показано, что в основе явления лежит захват свободных носителей - электронов на акцептороподобных изоэлектронных центрах $\mathrm{O}_{\mathrm{S}}$ с последующим образованием ассоциатов сложной структуры. Подтверждены предшествующие выводы о механизме растворения кислорода в $\mathrm{CdS}$ с отклонениями от стехиометрии. Изменения электрических свойств в активированном кислородом $\mathrm{PbS}(\mathrm{O})$, как и в $\mathrm{CdS}(\mathrm{O})$, показали, что изоэлектронные кислородные центры $\mathrm{O}_{\mathrm{S}}$ присутствуют в сульфиде свинца.

DOI: 10.21883/FTP.2018.03.45611.8493

\section{1. Введение}

В работах [1-6] рассматривались особенности растворения кислорода в соединениях $\mathrm{A}^{\mathrm{II}} \mathrm{B}^{\mathrm{VI}}$ с привлечением теории антипересекающихся зон (ВАС). Кислород как нейтральная примесь замещения в серных узлах решетки - $\mathrm{O}_{\mathrm{S}}$ является изоэлектронным акцептором (ИЭА) с предполагаемым эффективным зарядом. Вследствие большой разницы размеров и электроотрицательностей пара кислород-сера $\mathrm{O}-\mathrm{S}$ образует $\mathrm{O}_{\mathrm{S}}$ центр, который относится к HMAS (highly mismatched alloys). Новые представления позволяют предполагать наличие аналогичных изоэлектронных кислородных центров и в других сульфидах, содержащих кислород.

Такой центр может проявляться и при исследовании полупроводниковых структур, включающих сульфиды и кислород, однако этот вопрос пока не ясен. Давно известно, например, что для сульфида свинца при введении кислорода наблюдаются изменения, имеющие много общего с исследованными нами в соединениях $\mathrm{A}^{\mathrm{II}} \mathrm{B}^{\mathrm{VI}}[4]$. Для пары $\mathrm{O}-\mathrm{S}$ в сульфиде свинца кислород в серном узле решетки $\mathrm{O}_{\mathrm{S}}$ - тот же изоэлектронный акцептор. Такой центр предполагает возникновение новых локализованных уровней вблизи дна зоны проводимости и при незначительном увеличении концентрации примеси вызывает изменение зонной структуры, в частности, обусловливает нетривиальное уменьшение запрещенной зоны. В ряде работ [7-9 и др.] стабильно отмечается возникновение глубоких уровней $\sim 0.23$ эВ ниже дна зоны проводимости $\mathrm{PbS}$, которые связываются с возникающими центрами сенсибилизации кислородом.

С увеличением концентрации кислорода в $\mathrm{PbS}$ наблюдаются изменения в спектрах поглощения и фотопроводимости, край фундаментального поглощения $\mathrm{PbS}$ сме- щается в длинноволновую сторону. Аномальное увеличение с повышением температуры ширины запрещенной зоны $E_{g}(T)$ не рассматривалось в случае образования кислородной мультизоны, хотя в высокоэнергетической области за краем наблюдалась новая полоса поглощения и др.

Кроме того, в работе [7] было обнаружено, что активация кислородом способна существенно влиять на концентрацию подвижных носителей - электронов, определяющих проводимость кристаллитов $\mathrm{PbS}$. При этом кислород позволяет минимизировать концентрацию электронов до появления дырок. Процесс можно регулировать подбором температурно-временны́х режимов обработки кристаллов, т. е. состава $\mathrm{Pb}-\mathrm{S}-\mathrm{O}$.

Подобная взаимосвязь концентрации свободных носителей - электронов или проводимости от концентрации кислорода при исследовании соединений $\mathrm{A}^{\mathrm{II}} \mathrm{B}^{\mathrm{VI}}$ ранее нами не рассматривалась, как и механизм введения кислорода в кристалл. Данное сообщение восполняет этот пробел, дополняя опубликованные ранее материалы по $\mathrm{CdS}[1-7]$ и позволяет сравнить поведение $\mathrm{O}_{\mathrm{S}}$ центров в этих кристаллах и $\mathrm{PbS}$.

\section{2. Эксперимент и обсуждение}

\section{1. Сульфид кадмия}

Рассмотрим результаты исследования проводимости на группе монокристаллов $\mathrm{CdS}$, выращенных из паровой фазы по методу Маркова [10]. На рис. 1 представлена взаимосвязь проводимости и состава кристаллов при изменении собственных точечных дефектов. Методики исследования описаны в работах [3,5,11]. Результаты измерения проводимости образцов обозначены точками, 


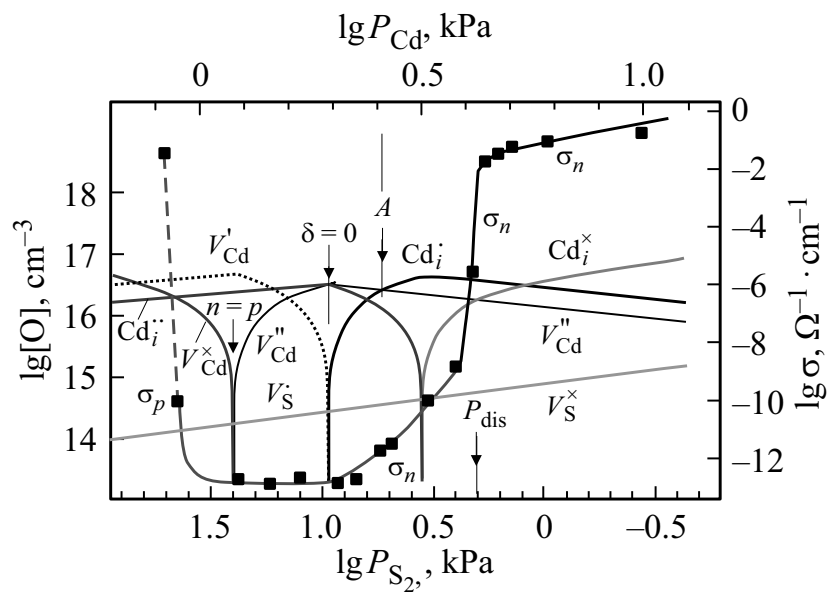

Рис. 1. Изменение экспериментально измеренной электрической проводимости $\sigma$ монокристаллов $\mathrm{CdS}(\mathrm{O})$ (точки) в пределах области гомогенности соединения сопоставлено с равновесной диаграммой дефектообразования, характеризующей изменение типа собственных дефектов (СТД) в CdS. Зарядовые состояния дефектов $(-,--, 0,+,++)$ обозначены как $\left({ }^{\prime},{ }^{\prime \prime}, \times, \bullet, \cdots\right)$ соответственно.

которые одновременно определяют состав кристалла по шкале давлений паров компонентов при росте. Диаграмма равновесия собственных точечных дефектов (СТД) рассчитана теоретически для чистого $\mathrm{CdS}$ при температуре $1100^{\circ} \mathrm{C}[3-5]$.

Сульфид кадмия имеет преимущественно $n$-тип проводимости. ${ }^{1}$ Межузельный кадмий $\mathrm{Cd}_{i}$ является превалирующим собственным донором решетки. Возникновение свободных носителей (электронов) в кристаллах обязано двум типам этих мелких доноров $-\mathrm{Cd}_{i}^{\times} 0.04$ эВ и $\mathrm{Cd}_{i}^{\bullet} \sim 0.1$ эВ [3].

При избытке кадмия преобладают доноры $\mathrm{Cd}_{i}^{\times(\bullet)} \mathrm{c}$ энергией ионизации $\sim 0.04$ эВ [3]. Они плавно убывают с ростом $P_{S 2}$, что согласуется с уменьшением проводимости $N$-типа $\sigma_{n}$ (рис. 1$)$.

При увеличении избытка серы $\lg P_{S_{2}} \geq P_{\text {dis }}$ появляются и начинают преобладать заряженные доноры второй ионизации $\mathrm{Cd}_{i}^{\bullet \bullet \bullet)} \sim 0.1$ эВ [3]. С увеличением их концентрации следовало ожидать роста проводимости, однако проводимость резко уменьшается.

Согласно диаграмме равновесия СТД (рис. 1) видно, что вблизи точки $P_{\text {dis }}$ ведущими становятся пары взаимно компенсирующих дефектов $\mathrm{Cd}_{i}$ и $V_{\mathrm{Cd}}^{\prime \prime}$. Уменьшение проводимости продолжается до $\lg P_{S_{2}} \approx 1$. Из детального рассмотрения рис. 1 видно, что ход кривой $\sigma_{n}$ зеркально отражает ход концентраций $\mathrm{Cd}_{i}$ в области стехиометрии от точки $P_{\text {dis }}$ до $\lg P_{S_{2}} \approx 0.9-1$. Поэтому можно полагать, что проводимость следует за концентрацией именно $\mathrm{Cd}_{i}^{\bullet}$ во всей этой области.

Из литературы хорошо известно, что дефект $\mathrm{Cd}_{i}^{\bullet}$ вводит кислород в $\mathrm{CdS}$ [4]. Связь растворимости кислорода

${ }^{1}$ В образцах с предельным избытком серы в некоторых работах наблюдался $p$-тип проводимости с появлением глубоких собственных акцепторов $V_{\mathrm{Cd}}^{\times}$(см. точку $n=p$ на диаграмме). с присутствием $\mathrm{Cd}_{i}^{\bullet}$ детально описана в работах [1-5]. Растворимость кислорода для исследуемых кристаллов в зависимости от условий роста из газовой фазы приведена на рис. 2 по данным [3-5].

Видно, что резкий спад проводимости вблизи точки $P_{\text {dis }}$ совпадает с увеличением растворимости кислорода в кристаллах. Одновременно, как мы уже отмечали, преобладающим типом дефектов становятся $\mathrm{Cd}_{i}$. Область повышенной концентрации этих дефектов соответствует области повышенного содержания растворенного кислорода $\left(\lg P_{S_{2}} \approx 0.3-1\right)$. Концентрация кислорода здесь порядка или меньше $10^{20} \mathrm{~cm}^{-3}$. Только в точке стехиометрии $\delta=0$, где концентрация $\left[\mathrm{Cd}_{i}^{\bullet}\right]$ спадает до нуля, концентрация кислорода уменьшается до уровня фона. В узкой области диаграммы $\lg P_{S_{2}} \approx 0.9$, когда дефекты $\mathrm{Cd}_{i}^{\bullet}$ еще присутствуют, сохраняется и растворенный кислород.

При этом область повышенного содержания растворенного кислорода $\left(\lg P_{S_{2}} \approx 0.3-1\right)$ соответствует области пониженной проводимости (рис. 1). Очевидно, что кислород является причиной уменьшения проводимости и это определяется захватом электронов изоэлектронным акцептором $\mathrm{O}_{\mathrm{S}}$.

В работах $[3,4]$ отмечалось, что введение кислорода приводит к образованию комплексов А-центров самоактивированного SA свечения CdS. На рис. 2 при $\left[\mathrm{Cd}_{i}^{\bullet}\right]=\left[V_{\mathrm{Cd}}^{\prime \prime}\right]$ точка $\mathrm{A}$ - это сравнительно узкая область вблизи максимальной $\left[\mathrm{O}_{\mathrm{S}}\right]$. Область повышенного содержания растворенного кислорода $\left(\lg P_{S_{2}} \approx 0.3-1\right)$ шире и менее четко выражена (рис. 1,2). Рассмотрим возможность образования комплексов в обоих случаях.

Захват кислородом электронов $e^{\prime}$ возможен только с доноров $\mathrm{Cd}_{i}^{\bullet}-e^{\prime} \rightarrow \mathrm{Cd}_{i}^{\bullet \bullet}$ с последующим взаимодействием заряженных центров $\mathrm{Cd}_{i}^{\bullet}$ и компенсирующего $V_{\mathrm{Cd}}^{\prime \prime}$ акцептора. Это предопределяет состав кислородосодержащего центра $\left\{V_{\mathrm{Cd}}^{\prime \prime}-\mathrm{Cd}_{i}^{\bullet \bullet}-\mathrm{O}_{\mathrm{S}}^{\prime}\right\}^{\prime}$ как однократно заряженного комплекса, подобно принятому ранее,

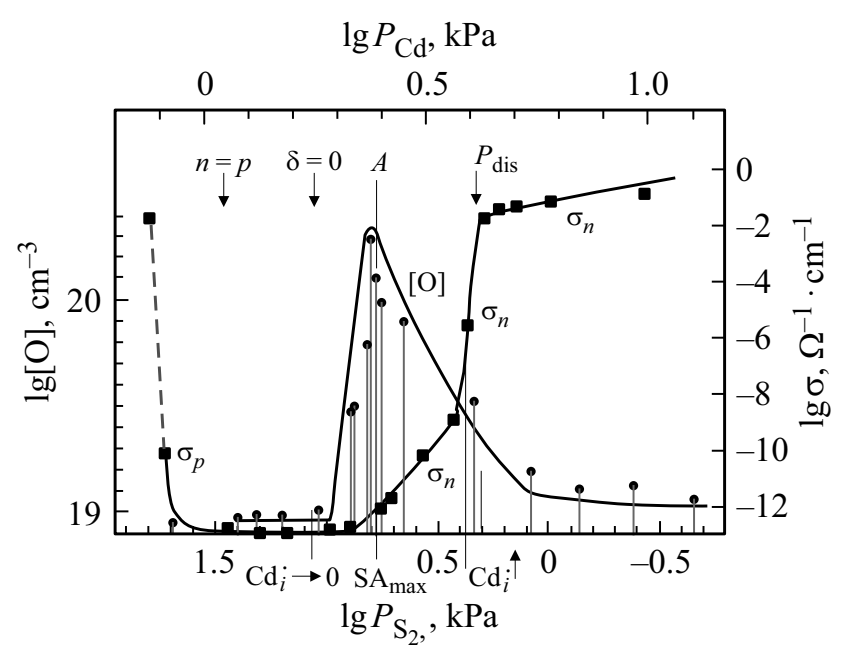

Рис. 2. Концентрации кислорода [3], представленные на штрихдиаграмме, определяются длиной штрихов. Проводимость $\sigma$ нанесена в соответствии с рис. 1 . 
который является рекомбинационным уровнем SA свечения $[1,2,5]$. Комбинация и число заряженных частиц в нем соответствует только составам точки А, когда концентрации $\mathrm{Cd}_{i}^{\bullet}$ и $V_{\mathrm{Cd}}^{\prime \prime}$ равны.

Для прочих составов области $\left(\lg P_{S_{2}} \approx 0.3-1\right)$ схема компенсации, согласно равновесию рис. 1, другая, в частности, $2\left[\mathrm{Cd}_{i}^{\bullet}\right]=\left[V_{\mathrm{Cd}}^{\prime \prime}\right]$. В этом случае вне описанного А-комплекса остаются дефекты: $\mathrm{Cd}_{i}^{\bullet}\left(-e^{\prime} \rightarrow \mathrm{Cd}_{i}^{\bullet \bullet}\right)$ и $\mathrm{O}_{\mathrm{S}}$ $\left(+e^{\prime} \rightarrow \mathrm{O}_{\mathrm{S}}^{\prime}\right)$, взаимодействие которых дает дополнительно другие ассоциаты.

Полученные результаты дополняют и уточняют опубликованные ранее данные $[1-5,11]$. Образование прочных кислородосодержащих комплексов определяет и увеличение растворимости кислорода, и связывание подвижных носителей, что является причиной уменьшения электронной проводимости $\mathrm{CdS}$.

\section{2. Сульфид свинца}

Изменение проводимости $n$-типа с введением кислорода в $\mathrm{CdS}$ согласуется с описанными выше изменениями концентрации электронов в сульфиде свинца после его „очувствления“" кислородом [7].

Очевидно, что после введения кислорода в сульфид свинца с образованием твердого раствора $\mathrm{PbS}(\mathrm{O})$ следует ожидать создания изоэлектронных $\mathrm{O}_{\mathrm{S}}$ центров и кислородосодержащих комплексов. Связывая электроны, они воздействуют на электрические свойства материала, что позволяет создавать ИК-датчики с максимальной чувствительностью.

Согласно данным работы [7], кислород позволяет управлять концентрацией свободных носителей заряда. Введение кислорода в исходный $\mathrm{PbS}$ с типичной электронной проводимостью и низким сопротивлением (кОм на квадрат) позволяет получать высокоомные (МОм) слои $\mathrm{PbS}(\mathrm{O})$ с высоким временем жизни носителей $[7,9]$. Это является следствием захвата электронов (минимизацией их концентрации) изоэлектронными кислородными ловушками - $\mathrm{O}_{\mathrm{S}}$. Подбором температурно-временны́х режимов обработки состав $\mathrm{PbS}(\mathrm{O})$ может быть переведен к $p$-типу проводимости с высоким временем жизни дырок.

Переход к $p$-типу проводимости при введении кислорода $[7,9]$ осуществляется при прохождении через область стехиометрии состава соединения. Аналогичные результаты описаны выше на $\mathrm{CdS}$. Кислородные уровни при этом стабильны в объеме кристаллитов сульфида свинца и не удаляются при всех обработках технологического цикла.

Полученные данные углубляют понимание механизмов воздействия изоэлектронных центров $\mathrm{O}_{\mathrm{s}}$ на подвижные носители и электрические свойства кристаллов сульфида свинца при легировании кислородом.

\section{3. Заключение}

Развитие исследований изоэлектронных центров $\mathrm{O}_{\mathrm{S}}$ на базе представлений сравнительно молодой теории ВАС приводит все новые факты, которые позволяют судить не только о присутствии и структуре самих центров, но и влиянии их на физико-химические свойства кристаллов.

Расширение базы полученных данных, в частности при изучении электрических свойств, делает более прозрачными как формирование кислородосодержащих комплексов с участием определенного типа СТД, так и процесс вхождения кислорода в кристалл.

Результаты данной работы уточняют структуру и состав кислородных центров в $\mathrm{CdS}$, в частности зарядовое состояние кислородосодержащего А-центра как однократно отрицательно заряженного комплекса.

Подтверждено различное поведение кислорода в кристаллах разного состава в пределах области стехиометрии.

Обнаружение особенностей $\mathrm{O}_{\mathrm{S}}$ центров, проявляющихся в сходных по свойствам материалах - $\mathrm{PbS}$, стимулирует исследования кислородных центров в них с позиций теоретически обоснованных представлений ВАС.

Работа проводилась при поддержке гранта РФФИ № 16-07-00417-а „Управление параметрами и характеристиками фоторезистивных структур на основе халькогенидов свинца“.

\section{Список литературы}

[1] Н.К. Морозова, А.А. Канахин, И.Н. Мирошникова. Матер. докл. 46-го Междунар. науч.-техн. сем. „Флуктуационные и деградационные процессы в полупроводниковых приборах“ (М., МЭИ, 2016) с. 72.

[2] N.K. Morozova, A.A. Kanakhin. Semiconductors, 50, 849 (2016).

[3] Н.Д. Данилевич. Автореф. канд. дис. (М., МЭИ, 2011).

[4] N.K. Morozova, D.A. Mideros, N.D. Danilevich. Oxygen in Optics of II-VI Compounds in the Light of the Theory of Anticrossing Zones (LAP, Saarbrucken, Germany, 2013) [in Russian].

[5] А.А. Канахин. Автореф. канд. дис. (М., МЭИ, 2015).

[6] H.P. Hjalmarson, P. Vogl, D.J. Wolford, J.D. Dow. Phys. Rev. Lett., 44 (12), 810 (1980).

[7] Б.Н. Мирошников. Автореф. канд. дис. (М., МЭИ, 2016).

[8] T.H. Johnson. J. Proc. SPIE, 443, (1984), 60-9.

[9] Ю.И. Равич, Б.А. Ефимова, Н.А. Смирнов. Методы исследования полупроводников в применении к халькогенидам свинца (М., Наука, Гл. ред. физ.-мат. лит., 1968).

[10] В.А. Теплицкий. Автореф. канд. дис. (М., МИЭТ, 1989).

[11] Physics and Chemistry of II-VI Compounds, ed. by M. Aven, J.S. Prener (North_Holland, Amsterdam, 1967; Mir, Moscow, 1970).

Редактор А.Н. Смирнов 


\section{Isoelectronic oxygen centers and conductivity of $\mathrm{CdS}$ in comparison with $\mathrm{PbS}$}

N.K. Morozova, B.N. Miroshnikov

National Research University

„Moscow Power Engineering Institute“, 111250 Moscow, Russia

Abstract Commonly known effect of oxygen on the electrical properties of $\mathrm{PbS}$. The aim of this study was the comparison and detection of this phenomenon on the single crystals of $\mathrm{CdS}(\mathrm{O})$, thoroughly investigated by us earlier. The experiments were performed on single crystals of CdS with a known oxygen concentration, deviation from stoichiometry and a certain set of intrinsic point defects. They are allowed to confirm the results described in of $\mathrm{PbS}$, and find out their nature. It is shown that the phenomenon is based on binding of free carriers - electrons at aktseptoropodobnyh centers $\mathrm{O}_{\mathrm{S}}$ with the subsequent formation of the associates complicated structure. Confirmed previous findings on the mechanism of oxygen dissolution in CdS with deviations from stoichiometry. The results presented interdependencies with conductivity and dissolved oxygen concentration allowed to explain the complicated changes in the electrical properties of the activated oxygen-PbS. Changes in the electrical properties of the activated oxygen $\operatorname{PbS}(\mathrm{O})$, as well as in $\operatorname{CdS}(\mathrm{O})$, have shown that the oxygen isoelectronic centers are present in lead sulfide. 